Atıf için / For Citation: F. D. Koparal, Y. Özdemir, "Sıklaştırma fonksiyonlu çizge-yönlendirilmiş yinelemeli fonksiyon sistemleri”, Süleyman Demirel Üniversitesi Fen Edebiyat Fakültesi Fen Dergisi, 15(1), 110-123, 2020.

\title{
Sıklaştırma Fonksiyonlu Çizge-Yönlendirilmiş Yinelemeli Fonksiyon Sistemleri
}

\author{
Fatma Diğdem KOPARAL ${ }^{1, *}$, Yunus ÖZDEMIR ${ }^{1}$ \\ ${ }^{1}$ Eskişehir Teknik Üniversitesi, Matematik Bölümü, Yunusemre Kampüsü, 26470, Eskişehir, Türkiye \\ *yazlşllan yazar e-posta: fdyildirim@eskisehir.edu.tr
}

(Alını̧ / Received: 13.09.2019, Kabul / Accepted: 17.04.2020, Yayımlanma / Published: 31.05.2020)

\begin{abstract}
Özet: Yinelemeli fonksiyon sistemleri (YFS) fraktal geometrinin önemli araçlarından biridir. Çizge-yönlendirilmiş yinelemeli fonksiyon sistemleri (ÇYFS) ise klasik yinelemeli fonksiyon sistemlerininin bir genelleştirmesi olarak literatürde yer almaktadır. Diğer taraftan, sonlu sayıda büzülme dönüşümü ve bir sıklaştırma dönüşümünden (sabit dönüşüm) oluşan sıklaştırma fonksiyonlu yinelemeli fonksiyon sistemleri de bir başka önemli genelleştirme olarak karşımıza çıkmaktadır. Bu çalışmada ise, sıklaştırma fonksiyonlu çizge-yönlendirilmiş yinelemeli fonksiyon sistemleri, klasik sıklaştırma fonksiyonlu yinelemeli fonksiyon sistemlerinin bir genellemesi olarak tanımlanmış ve bu yeni kavram örneklendirilmiştir. Ayrıca tanımlanan sıklaştırma fonksiyonlu çizge-yönlendirilmiş sistemin atraktörlerinin Hausdorff boyutları üzerinde durulmuştır. Bir sıklaştırma fonksiyonlu YFS'nin atraktörünün Hausdorff boyutu için klasik durumda var olan bir takım sonuçların, bu yeni tanımlanan sistemlerin atraktörleri için de geçerli olduğu gösterilmiştir.
\end{abstract}

Anahtar kelimeler: Hausdorff boyut, Sıklaştırma Fonksiyonu, Yinelemeli Fonksiyon Sistemi (YFS), Çizge-yönlendirilmiş YFS.

\section{Graph-Directed Iterated Function Systems with Condensation}

\begin{abstract}
The notion of (classical) iterated function system (IFS) is one of the important tools in fractal geometry. Graph-directed iterated function system (GIFS) is a well-known generalization of the classical IFS. However, "iterated function system with condensation (or inhomogeneous IFS)" which consists of finite number of contractions and a condensation map can also be considered as another generalization of IFS. In this study, mainly, we define the notion of "graph-directed iterated function system with condensation (GIFS with condensation)" as a generalization of the classical "iterated function system with condensation", and give an example. Moreover, we investigate the Hausdorff dimensions of the attractors of this new system. And by using the existing results of the classical case, we obtain similar results for the attractors of a GIFS with condensation.
\end{abstract}

Keywords: Hausdorff dimension, Condensation map, Iterated function system (IFS), Graph-directed IFS. 


\section{Giriş}

Fraktal geometride önemli rol oynayan ve yinelemeli fonksiyon sistemlerinin (YFS) atraktörleri olarak karşımıza çıkan kendine benzer kümeler, fraktalların önemli bir kısmını oluşturmaktadır. Sierpinski Üçgeni, Cantor Kümesi, Koch Eğrisi vb. bir çok klasik fraktal bir yinelemeli fonksiyon sisteminin atraktörü olarak elde edilmektedir ([1,3]). Bununla birlikte, bir tam metrik uzay ve üzerinde tanımlı sonlu tane büzülme dönüşümünün belirlemiş olduğu yinelemeli fonksiyon sistemlerinin bir genelleştirilmesi olarak, sonlu tane (birbirinden farklı olmasında sakınca olmayan) tam metrik uzay ve bu metrik uzaylar arasındaki bir takım büzülme dönüşümlerinden oluşan çizge-yönlendirilmiş yinelemeli fonksiyon sistemleri (ÇYFS) de literatürde yerini almıştır ([4,5]). Klasik YFS kavramının sabit bir kompakt küme ile zenginleştirilmesi olarak düşünülebilecek sıklaştırma fonksiyonlu (veya yoğunlaştırılmış) yinelemeli fonksiyon sistemleri de bu teoride önemli bir yere sahiptir [1,6].

Öte yandan, bahsi geçen sistemlerin atraktörleri olarak karşımıza çıkan kümelerin fraktal boyutlarının belirlenmesi fraktal geometride ve hatta uygulama alanı bulunan birçok alanda son derece önemli bir konudur. Bir YFS'nin atraktörünün fraktal boyutu (Hausdorff boyutu, Minkowski boyutu vb.) eğer ilgili YFS bir takım koşulları sağlarsa, (Moran denklemi olarak bilinen) bir denklemin tek gerçel kökü olarak hesaplanabilmektedir. Aksi halde ilgili boyutu hesaplamak oldukça zordur. Bunun yanında herhangi bir kümenin yaklaşık olarak fraktal boyutunu hesaplamak bile uygulama bulduğu alanlar açısından önem arz etmektedir $([1,7,8])$. Bir sıklaştırma fonksiyonlu YFS sisteminin atraktörünün Hausdorff boyutu ile ilgili literatürde ciddi çalışmalar mevcuttur $[9,10,11]$. Bir ÇYFS verildiğinde, bu sistemin atraktörlerinin (her birinin eşit olduğunu bildiğimiz) Hausdorff boyutları ile ilgili de elde edilmiş net sonuçlar bulunmaktadır $[4,12,13]$.

$\mathrm{Bu}$ bölümde yukarıda tanımlanmış kavramlar hakkında temel bilgi ve bazı örnekler verildikten sonra, 2. Bölümde sıklaştırma fonksiyonlu yinelemeli fonksiyon sistemlerinin bir genelleştirmesi olarak "sıklaştırma fonksiyonlu çizge-yönlendirilmiş yinelemeli fonksiyon sistemleri” tanımlanmıştır. 3. Bölümde ise tanımlanan bu yeni sistemin atraktörlerinin Hausdorff boyutları ile ilgili literatürde var olan bir takım sonuçlara benzer sonuçlar elde edilmiştir.

\subsection{Yinelemeli Fonksiyon Sistemleri}

$(X, d)$ tam metrik uzay ve $f_{1}, f_{2}, \ldots, f_{n}$ fonksiyonları da bu metrik uzay üzerinde $f_{i}: X \rightarrow X$ şeklinde büzülme katsayıları $r_{i}$ olan büzülme dönüşümleri olmak üzere, $\left\{X ; f_{1}, f_{2}, \ldots, f_{n}\right\}$ sistemine (büzülme katsayısı $r_{i}$ 'lerin maksimumu olan) bir (hiperbolik) yinelemeli fonksiyon sistemi (YFS) denir. Bu formda bir YFS verildiğinde, $X$ 'in boştan farklı tüm kompakt altkümelerinden oluşan ve $d$ 'nin belirlediği $h$ Hausdorff metriği ile tek türlü belirlenen ve tam olan $\mathcal{H}(X)$ kümesi üzerinde, $F: \mathcal{H}(X) \rightarrow \mathcal{H}(X), B \in \mathcal{H}(X)$ için

$$
F(B)=\bigcup_{i=1}^{n} f_{i}(B)
$$

şeklinde tanımlanan $F$ dönüşümü bir büzülme dönüşümü olup, sabit nokta teoreminden bu dönüşümün sabit noktası vardır ve tektir. Bu sabit nokta herhangi bir $B \in \mathcal{H}(X)$ için 
$B, F(B), \ldots, F^{n}(B), \ldots$ dizisinin (Hausdorff metriğine göre) limiti olarak elde edilir ([3]). İşte bu sabit noktaya, ilgili YFS'nin atraktörü (veya çekicisi) denir. Eğer bir $A$ kümesi bir YFS'nin atraktörü ise, yani $F(A)=A$ ise, bu kümenin kendi bir takım büzülmüş kopyalarının bir birleşimi olarak ifade edildiğine dikkat ediniz.

Örnek 1.1 $\left(\mathbb{R}^{2}, d_{s t d}\right)$ tam metrik uzayı üzerinde büzülme katsayıları $r_{i}=\frac{1}{2}$ olan $f_{i}: \mathbb{R}^{2} \rightarrow$ $\mathbb{R}^{2}(i=1,2,3)$

$$
f_{1}(x, y)=\left(\frac{x}{2}, \frac{y}{2}\right), f_{2}(x, y)=\left(\frac{x+1}{2}, \frac{y}{2}\right), f_{3}(x, y)=\left(\frac{x+1 / 2}{2}, \frac{y+\sqrt{3} / 2}{2}\right)
$$

(benzerlik) dönüşümlerinden oluşan $\left\{\mathbb{R}^{2} ; f_{1}, f_{2}, f_{3}\right\}$ sisteminin atraktörü Sierpinski üçgenidir (bkz. Şekil 1). Gerçekten de $S$ Sierpinski üçgenini göstermek üzere

şeklindedir.

$$
S=F(S)=\bigcup_{i=1}^{3} f_{i}(S)
$$
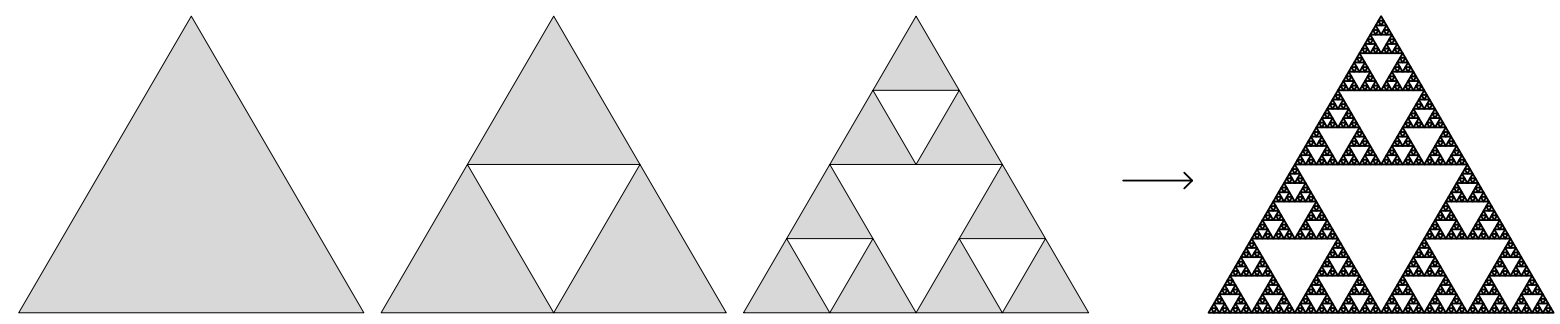

Şekil 1. $B$ (düzlemde sol alt köşesi orijinde olan) içi dolu eşkenar üçgen olmak üzere, $F^{n}(B)$ dizisinin Hausdorff limiti olarak Sierpinski üçgeni.

\subsection{Stklaşturma Fonksiyonlu Yinelemeli Fonksiyon Sistemleri}

$\left\{X ; f_{1}, f_{2}, \ldots, f_{n}\right\}$ şeklinde bir YFS verildiğinde, bu sisteme sabit bir $C \in \mathcal{H}(X)$ için $w: \mathcal{H}(X) \rightarrow \mathcal{H}(X), w(B)=C$ şeklinde bir sabit fonksiyon eklendiğinde elde edilen $\left\{X ; f_{1}, f_{2}, \ldots, f_{n}, w\right\}$ sistemi $w$ sıklaştırma fonksiyonlu bir YFS adını alır [2].

$F^{C}: \mathcal{H}(X) \rightarrow \mathcal{H}(X), B \in \mathcal{H}(X)$ için

$$
F^{C}(B)=w(B) \cup \bigcup_{i=1}^{n} f_{i}(B)
$$

şeklinde tanımlanan $F^{C}$ dönüşümü büzülme dönüşümü olmaya devam eder. Yine sabit nokta teoreminden bu dönüşümün sabit noktası vardır ve tektir. İlgili sabit noktaya verilen sıklaştırma fonksiyonlu yinelemeli fonksiyon sisteminin (SFYFS) atraktörü denir. Burada yapılan şey, var olan bir YFS'ni sabit bir küme ile sıklaştırmaktır.

Örnek 1.2. Örnek $1.1^{\prime}$ de verilmiş olan $\left\{\mathbb{R}^{2} ; f_{1}, f_{2}, f_{3}\right\}$ yinelemeli fonksiyon sistemine 
$C=\left\{(x, y) \mid x=\frac{1}{2},-1 \leq y \leq 0\right\} \quad$ olmak üzere $\quad w: \mathcal{H}\left(\mathbb{R}^{2}\right) \rightarrow \mathcal{H}\left(\mathbb{R}^{2}\right), w(B)=C$ fonksiyonunu ekleyelim. Bu durumda $\left\{\mathbb{R}^{2} ; f_{1}, f_{2}, f_{3}, w\right\}$ SFYFS'nin atraktörü Şekil 2'de verilmiştir.
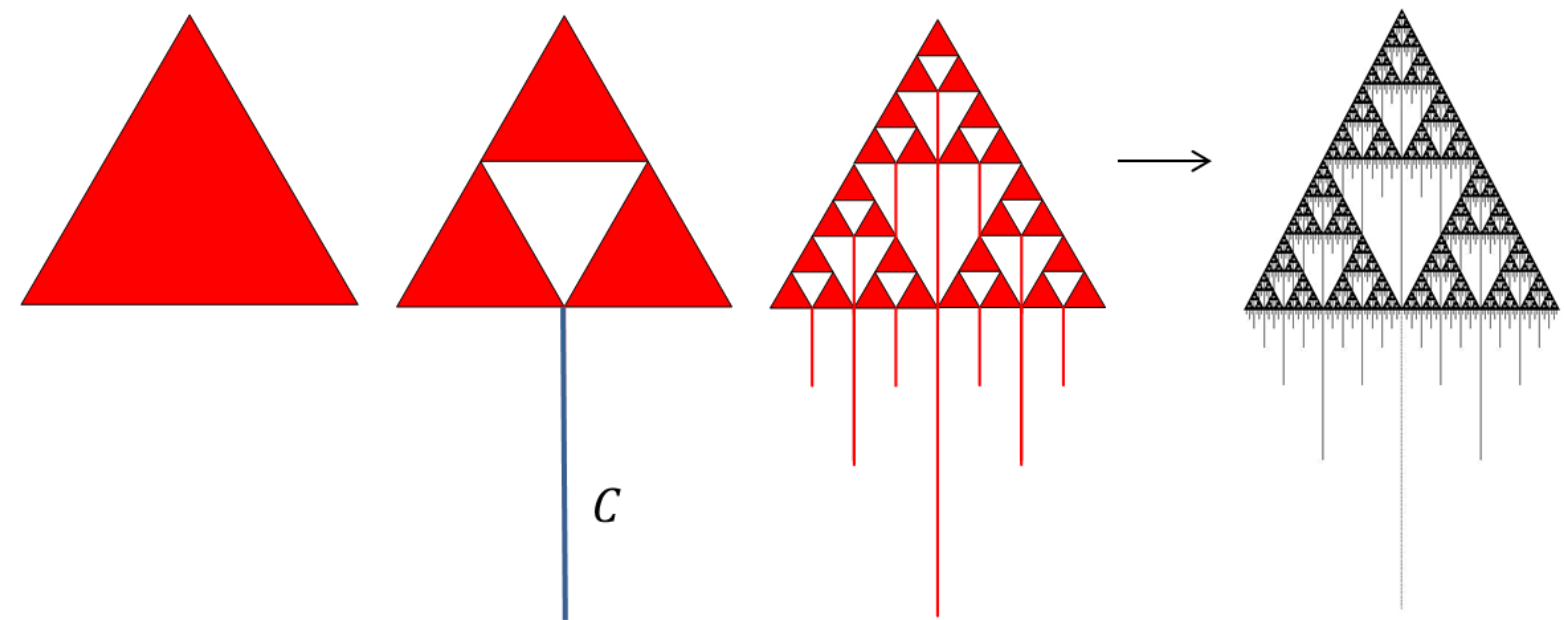

Şekil 2. $B$ (düzlemde sol alt köșesi orijinde olan) içi dolu eşkenar üçgen olmak üzere, $F^{C}$ dönüşümü altında $B$ kümesinin ilk iki iterasyonu ve bu iterasyonların sonucu elde edilen atraktör.

\section{3 Çizge-Yönlendirilmiş Yinelemeli Fonksiyon Sistemleri}

Daha teknik olarak, $u=1,2, \ldots, N$ olmak üzere $\left(X^{u}, d_{u}\right)$ tam metrik uzayları ve bu metrik uzaylar arasında büzülme katsayıları $r_{j}^{u v}$ olan

$$
f_{\mathrm{j}}^{u v}: X^{v} \rightarrow X^{u}\left(u, v=1,2, \ldots, N, j=1,2, \ldots, K^{u v}\right)
$$

fonksiyonları verilmiş olsun (burada $K^{u v}$ ilgili metrik uzaylar arasındaki fonksiyon sayısını göstermektedir). Bu durumda $\left\{X^{u} ; f_{j}^{u v}, u, v=1,2, \ldots, N, j=1,2, \ldots, K^{u v}\right\}$ (ya da kisaca $\left\{X^{u} ; f_{j}^{u v}\right\}$ ) sistemine çizge-yönlendirilmiş yinelemeli fonksiyon sistemi (ÇYFS) denir. $N>1$ ve en az bir $u \neq v$ çifti için $K^{u v}>0$ olduğunu kabul edelim.

Yardımcı Teorem 1.1. $\left\{X^{u} ; f_{j}^{u v}\right\}$ çizge-yönlendirilmiş YFS verilmiş olsun. Bu durumda,

olmak üzere

$$
F_{u}: \mathcal{H}\left(X^{1}\right) \times \cdots \times \mathcal{H}\left(X^{N}\right) \rightarrow \mathcal{H}\left(X^{u}\right), F_{u}(B)=F_{u}\left(B^{1}, B^{2}, \ldots, B^{N}\right)=\bigcup_{v=1}^{N} \bigcup_{j=1}^{K^{u v}} f_{j}^{u v}\left(B^{v}\right)
$$

$$
F: \mathcal{H}\left(X^{1}\right) \times \cdots \times \mathcal{H}\left(X^{N}\right) \rightarrow \mathcal{H}\left(X^{1}\right) \times \cdots \times \mathcal{H}\left(X^{N}\right), F(B)=\left(F_{1}(B), \ldots, F_{N}(B)\right)
$$

şeklinde tanımlanan fonksiyon (büzülme katsayısı $\max r_{j}^{u v}$ olan) bir büzülme dönüşümüdür ve yine sabit nokta teoreminden bu dönüşümün her $u=1,2, \ldots, N$ için 


$$
A^{u}=\bigcup_{v=1}^{N} \bigcup_{j=1}^{K^{u v}} f_{j}^{u v}\left(A^{v}\right)
$$

koşulunu sağlayan (boştan farklı kompakt kümelerden oluşan) ve atraktör (sistemi) olarak adlandırılan $\left(A^{1}, A^{2}, \ldots, A^{N}\right)$ sabit noktası vardır [4].

Sonuçta atraktör (sistemi) olarak her biri kendisinin ve/veya diğerlerinin bir takım büzülmüşlerinin bir birleşimi olarak ifade edilen $\left\{A^{u} \mid u=1,2, \ldots, N\right\}$ kompakt kümeler elde edilir. Burada ilgili $F$ fonksiyonunun, çarpım uzayı üzerindeki $h_{\max }$ maksimum metriği (kısalık için bu aşamadan sonra $h$ ile gösterilecektir) ile bir tam metrik uzay olan $\mathcal{H}\left(X^{1}\right) \times \cdots \times \mathcal{H}\left(X^{N}\right)$ üzerinde tanımlandığını hatırlatmış olalım (Her bir $u$ için $\mathcal{H}\left(X^{u}\right)$, $h_{u}$ Hausdorff metriği ile bir tam metrik uzaydır).

Örnek 1.3. $X^{1}$ ve $X^{2}$ metrik uzaylarını standart metrikle tam olan $\mathbb{R}^{2}$ olarak alalım. Şekil 3'te verilen $A \subset X^{1}=\mathbb{R}^{2}$ birim karesini ve $B \subset X^{2}=\mathbb{R}^{2}$ ikizkenar üçgeni düşünelim. $X^{1}$ ve $X^{2}$ metrik uzayları arasındaki (benzerlik dönüşümü olan) büzülme dönüşümleri $A$ ve $B$ yardımıyla Şekil 4 'te resmedilmiştir. Burada $X^{1}$ 'den $X^{1}$ 'e 4 tane 1/3'lük, $X^{2}$ 'den $X^{1}$ 'e 4 tane $1 / 3 \sqrt{2}$ 'lik, $X^{1}$ 'den $X^{2}$ 'ye 1 tane $2 / 5$ 'lik ve son olarak $X^{2}$ 'den $X^{2}$ 'ye 2 tane $1 / 2$ 'lik benzerlik dönüşümü vardır. Bu sistemin belirlediği atraktörler Şekil 5 'te gösterilmiştir.
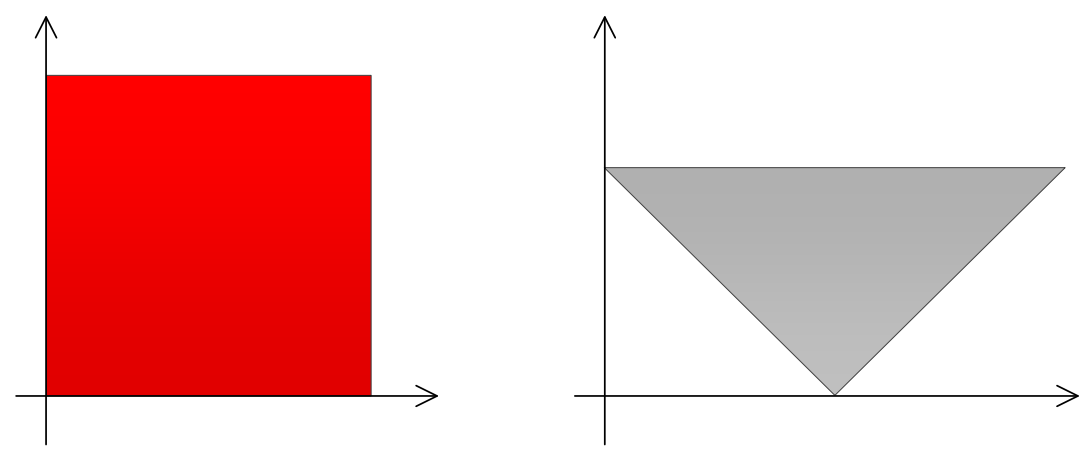

Şekil 3. $A$ birim karesi (solda) ve dik kenarları 1 birim uzunluğunda olan $B$ ikizkenar dik üçgeni (sağda).
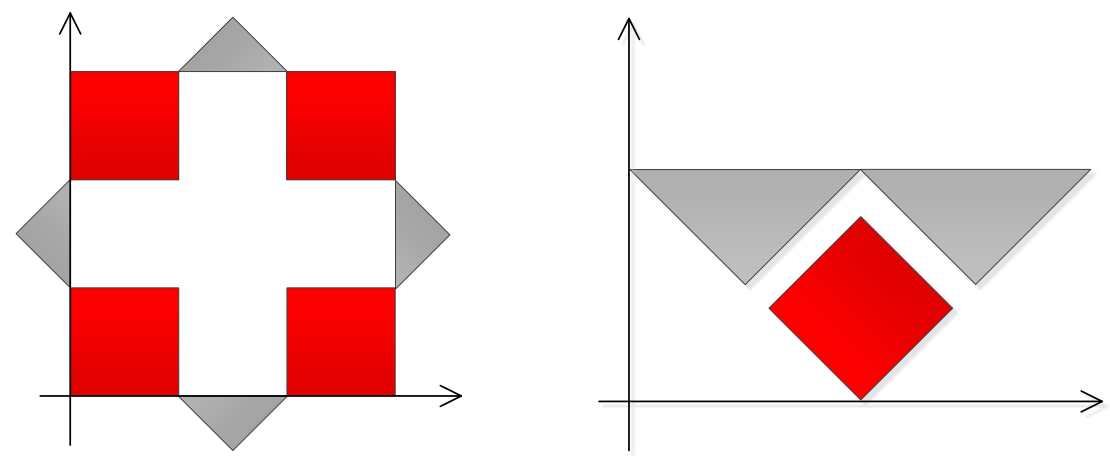

Şekil 4. Metrik uzaylar arasındaki büzülme dönüşümlerinin tasviri. 

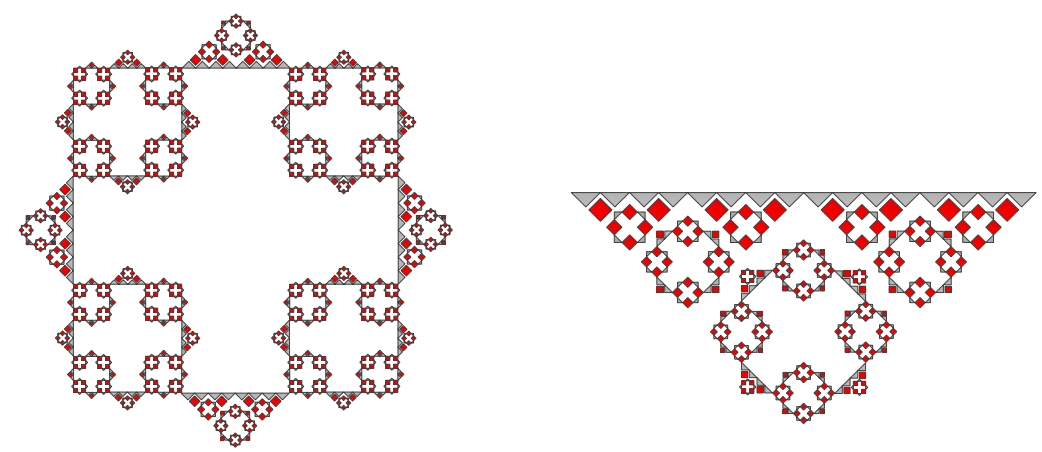

Şekil 5. İterasyonun dördüncü adımında ilgili ÇYFS'nin atraktörleri: $A^{1}$ (solda), $A^{2}$ (sağda).

$\left\{X^{u} ; f_{\mathrm{j}}^{u v}, u, v=1, \ldots, N, j=1, \ldots, K^{u v}\right\}$ şeklinde bir ÇYFS, tek türlü olarak bir yönlendirilmiş ve ağırlıklı çizge belirler, şöyle ki; her bir metrik uzayı bir köşe ve sistemdeki herhangi bir $f_{\mathrm{j}}^{u v}$ fonksiyonunu da $v$ köşesinden $u$ köşesine bir (yönlü) kenar olarak alalım. Bu durumda elimizde $N$ köşeli yönlendirilmiş bir çizge olur. Bu nedenle bu sistem çizge-yönlendirilmiş YFS adını alır. Hatta her bir kenar üzerine ilgili fonksiyonun büzülme katsayısını ağırlık olarak eklersek, bir ÇYFS tek türlü bir ağırlıklı yönlendirilmiş çizge belirler (Şekil 6'da Örnek 1.3'de verilen ÇYFS'nin çizgesi verilmiştir). Bu nedenle ilgili sistemi şu şekilde de ifade edebiliriz: Bir çizge yönlendirilmiş YFS, $V$ köşe noktalarını, $E$ tüm (yönlü) kenarların kümesini, $E_{u v} u$ 'dan $v$ 'ye giden kenarların kümesini, $E_{u} u$ 'dan çıkan kenarların kümesini ve $r(e)$ de $e \in E_{u v}$ kenarına ilişkin $f_{e}: X^{v} \rightarrow X^{u}$ fonksiyonunun büzülme katsayısını göstermek üzere $\mathcal{G}=(V, E, r(e))$ çizgesi ile temsil edilebilir. Bu yeni gösterimlerle de bu sistemin atraktörü $\left(A^{u}\right)_{u \in V}$ şeklinde gösterilir ve $\forall u \in V$ için

$$
A^{u}=\bigcup_{v \in V} \bigcup_{e \in E_{u v}} f_{e}\left(A^{v}\right)
$$

şeklindedir.

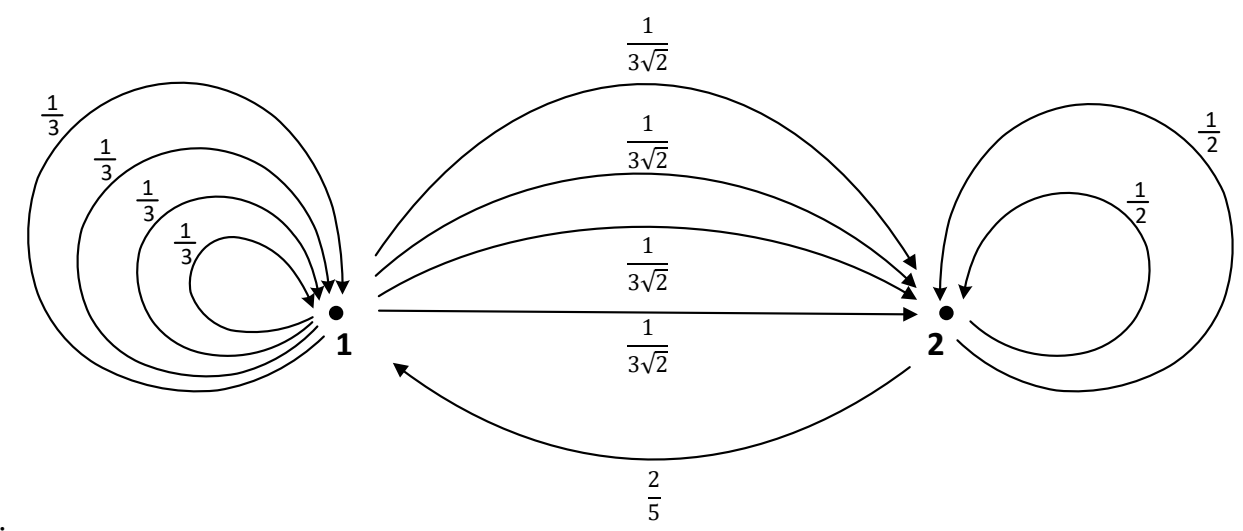

Şekil 6. Örnek 1.3'de verilen ÇYFS'nin gerçeklediği Mauldin-Williams çizgesi (Yönlü kenarlar üzerindeki sayılar ilgili kenarın ağırlığını, yani kenarı belirleyen büzülmenin benzerlik katsayısını göstermektedir). 
Bu noktadan sonra, $\mathcal{G}=(V, E, r(e))$ çizgesini gerçekleyen bir ÇYFS, kısaca $\left\{X^{u} ;\left(f_{e}\right)_{e \in E}\right\}$ şeklinde gösterilecektir.

Yönlendirilmiş ağırlıklı bir $\mathcal{G}=(V, E, r(e))$ çizgesinde $m$ uzunluklu bir yolu, $e_{i} \in E$ ve $e_{i}$ 'nin bitiş noktası $e_{i+1}$ 'in başlangıç noktası olmak üzere $\alpha=e_{1} e_{2} \ldots e_{m}$ şeklinde gösterelim (bu yolun uzunluğu içerdiği kenar sayısı kadardır ve $|\alpha|=\mathrm{m}$ şeklinde gösterilir). $i(\alpha) \alpha$ yolunun başlangıç köşesini $\left(e_{1}\right)$ göstersin. Eğer her $u$ için $E_{u} \neq \emptyset$ ise $\mathcal{G}$ çizgesine bir Mauldin-Williams çizgesi denir [5]. Ayrıca eğer herhangi bir $u$ köşesinden bir $v$ köşesine bir (yönlü) yol bulunabiliyorsa, bu türden çizgelere kuvvetli-bağlantılı çizge denir.

\section{Sıklaştırma Fonksiyonlu Çizge-Yönlendirilmiş YFS}

$\mathrm{Bu}$ çalışmanın temel amacı olarak, çizge-yönlendirilmiş bir yinelemeli fonksiyon sisteminde, her bir tam metrik uzay (veya köşe) için bir sıklaştırma kümesi yani bir sabit fonksiyon sisteme eklenerek klasik durumun bir genellemesi olarak sıklaştırma fonksiyonlu çizge-yönlendirilmiş YFS (SÇYFS) kavramı tanımlanacaktır. Bu bölümde, bahsedilen genellemenin mümkün olduğu kanıtlanıp örneklendirilecek ve son bölümde ise bu yeni tipteki ÇYFS atraktörlerinin Hausdorff boyutu ile ilgili klasik durumunda var olan sonuca benzer bir sonuç elde edilecektir.

Tanım 2.1 $\left\{X^{u} ;\left(f_{e}\right)_{e \in E}\right\} \quad$ sistemi $\mathcal{G}=(V, E, r(e)) \quad$ Mauldin-Williams çizgesini gerçekleyen bir ÇYFS olsun. Her $u \in V$ için $C^{u} \in \mathcal{H}\left(X^{u}\right)$ sabit bir kompakt küme olmak üzere

$$
w_{u}: \mathcal{H}\left(X^{u}\right) \rightarrow \mathcal{H}\left(X^{u}\right), w_{u}(B)=C^{u}\left(B \in \mathcal{H}\left(X^{u}\right)\right)
$$

şeklinde tanımlanan fonksiyona sıklaştırma fonksiyonu, $C^{u}$ kümesine de sıklaştırma kümesi denir. $\left\{X^{u} ;\left(f_{e}\right)_{e \in E}, w_{u}\right\}$ sistemine de sıklaştırma fonksiyonlu çizge-yönlendirilmiş yinelemeli fonksiyon sistemi (SÇYFS) adı verilir.

Yardımcı Teorem $2.1\left\{X^{u} ;\left(f_{e}\right)_{e \in E}\right\}$ sistemi $\mathcal{G}=(V, E, r(e))$ Mauldin-Williams çizgesini gerçekleyen bir ÇYFS olmak üzere $\left\{X^{u} ;\left(f_{e}\right)_{e \in E}, w_{u}\right\}$ SÇYFS verilsin. Bu durumda $\boldsymbol{B}=\left(B^{v}\right)_{v \in V} \in \prod_{v \in V} \mathcal{H}\left(X^{v}\right)$ için

$$
F_{u}(\boldsymbol{B})=\bigcup_{v \in V} \bigcup_{e \in E_{u v}} f_{e}\left(B^{v}\right) \text { ve } F_{u}^{C}(\boldsymbol{B})=w_{u}\left(B^{u}\right) \cup F_{u}(\boldsymbol{B})
$$

olmak üzere çarpım uzayı üzerinde

$$
F^{C}: \prod_{v \in V} \mathcal{H}\left(X^{v}\right) \rightarrow \prod_{v \in V} \mathcal{H}\left(X^{v}\right), F^{C}(\boldsymbol{B})=\left(F_{v}^{C}(\boldsymbol{B})\right)_{v \in V}
$$

olarak tanımlanan $F^{C}$ dönüşümü büzülme katsayısı $r=\max _{e \in \mathrm{E}} r(e)$ olan büzülme dönüşümüdür.

Kanıt. $F^{\prime}$ nin bir büzülme dönüşümü olduğunu göstermek için, $\boldsymbol{A}=\left(A^{v}\right)_{v \in V}, \boldsymbol{B}=\left(B^{v}\right)_{v \in V}$ olmak üzere 


$$
h\left(F^{C}(\mathbf{A}), F^{C}(\boldsymbol{B})\right) \leq r \cdot h(\boldsymbol{A}, \boldsymbol{B})
$$

eşitsizliğini sağlayan bir $0<r<1$ sayısının var olduğu gösterilmelidir. $\prod_{v \in V} \mathcal{H}\left(X^{v}\right)$ üzerindeki $h=h_{\max }$ metriğinin tanımından

$$
h\left(F^{C}(\mathbf{A}), F^{C}(\boldsymbol{B})\right)=\max _{u \in V}\left\{h_{u}\left(F_{u}^{\mathrm{C}}(\boldsymbol{A}), F_{u}^{\mathrm{C}}(\boldsymbol{B})\right)\right\}
$$

şeklindedir. Ayrıca herhangi bir $(X, d)$ tam metrik uzayında $A, B, C, D \in \mathcal{H}(X)$ için, $h_{0}$ $\mathcal{H}(X)$ üzerindeki Hausdorff metriğini göstermek üzere

$$
h_{0}(A \cup B, C \cup D) \leq \max \left\{h_{0}(A, C), h_{0}(B, D)\right\}
$$

eşitliği geçerlidir. Tanımdan ve yukarıdaki özellikten

$$
\begin{aligned}
h\left(F^{C}(\mathbf{A}), F^{C}(\boldsymbol{B})\right) & =\max _{u \in V}\left\{h_{u}\left(F_{u}^{\mathrm{C}}(\boldsymbol{A}), F_{u}^{\mathrm{C}}(\boldsymbol{B})\right)\right\} \\
& =\max _{u \in V}\left\{h_{u}\left(w_{u}\left(A^{u}\right) \cup F_{u}(\boldsymbol{A}), w_{u}\left(B^{u}\right) \cup F_{u}(\boldsymbol{B})\right)\right\} \\
& \leq \max _{u \in V}\left\{\max \left\{h_{u}\left(F_{u}(\boldsymbol{A}), F_{u}(\boldsymbol{B})\right), h_{u}\left(w_{u}\left(A^{u}\right), w_{u}\left(B^{u}\right)\right)\right\}\right\} \\
& =\max _{u \in V}\left\{\max \left\{h_{u}\left(F_{u}(\boldsymbol{A}), F_{u}(\boldsymbol{B})\right), h_{u}\left(C^{u}, C^{u}\right)\right\}\right\} \\
& =\max _{u \in V}\left\{h_{u}\left(F_{u}(\boldsymbol{A}), F_{u}(\boldsymbol{B})\right)\right\}
\end{aligned}
$$

ve, Yardımcı Teorem 1.1'den $r=\max r_{j}^{u v}$ olmak üzere $h\left(F^{C}(\mathbf{A}), F^{C}(\boldsymbol{B})\right) \leq r \cdot h(\boldsymbol{A}, \boldsymbol{B})$ elde edilmiş olur.

Sabit nokta teoreminden, bu şekilde verilen bir sistemin de yine tek türlü belirli ve adına sistemin atraktörü (veya atraktör sistemi) diyeceğimiz kompakt kümelerden oluşan $\left(A^{u}\right)_{u \in V}$ kompakt küme ailesi vardır.

Teorem 2.2 $\left\{X^{u} ;\left(f_{e}\right)_{e \in E}\right\} \quad$ sistemi $\mathcal{G}=(V, E, r(e))$ Mauldin-Williams çizgesini gerçekleyen bir ÇYFS olmak üzere $\left\{X^{u} ;\left(f_{e}\right)_{e \in E}, w_{u}\right\}$ SÇYFS verilsin. Bu durumda her $u \in$ $V$ için

$$
A^{u}=C^{u} \cup \bigcup_{v \in V} \bigcup_{e \in E_{u v}} f_{e}\left(A^{v}\right)
$$

eşitliğini sağlayan boş kümeden farklı tek türlü belirli $\left\{A^{u} \subseteq X^{u} \mid u \in V\right\}$ kompakt küme ailesi vardır.

Kanıt. Her $u \in V$ için $\mathcal{H}\left(X^{u}\right)$ 'nun üzerindeki $h_{u}$ Hausdorff metriği ile bir tam metrik uzaydır. Bu durumda $\prod_{v \in V} \mathcal{H}\left(X^{v}\right)$ çarpım uzayı da 


$$
\boldsymbol{A}=\left(A^{v}\right)_{v \in V}, B=\left(B^{v}\right)_{v \in V} \in \prod_{v \in V} \mathcal{H}\left(X^{v}\right)
$$

olmak üzere,

$$
h(\boldsymbol{A}, \boldsymbol{B})=\max _{u \in V}\left\{h_{u}\left(A^{u}, B^{u}\right)\right\}
$$

şeklinde tanımlı maximum metrikle bir tam metrik uzaydır.

Öte yandan, bu çarpım uzayı üzerinde tanımlı $F^{C}$ dönüşümünün bir büzülme dönüşümü olduğu Yardımcı Teorem 2.1'de gösterilmiştir.

Sonuç olarak, bu dönüşümün Banach Sabit Nokta Teoremi gereğince, her $u \in V$ için

$$
F^{c}\left(A^{u}\right)=A^{u}=C^{u} \cup \bigcup_{v \in V} \bigcup_{e \in E_{u v}} f_{e}\left(A^{v}\right)
$$

olacak şekilde boş kümeden farklı tek türlü belirli $\left\{A^{u} \subseteq X^{u} \mid u \in V\right\}$ kompakt küme ailesi vardır ve tektir.

Örnek 2.1 Örnek 1.3'de verilen ÇYFS'ni düşünelim. Bu ÇYFS'ne $u=1,2$ için Şekil 7'de belirtilen kompakt kümelerin belirlediği ilgili $w_{v}: \mathcal{H}\left(\mathbb{R}^{2}\right) \rightarrow \mathcal{H}\left(\mathbb{R}^{2}\right)$ siklaştırma dönüşümlerinin eklenmesi ile oluşan sıklaştırma fonksiyonlu ÇYFS'nin (Şekil 8'de sistemdeki tüm dönüşümler şekilsel olarak tarif edilmiştir.) atraktörleri de Şekil 9'da gösterilmiştir.
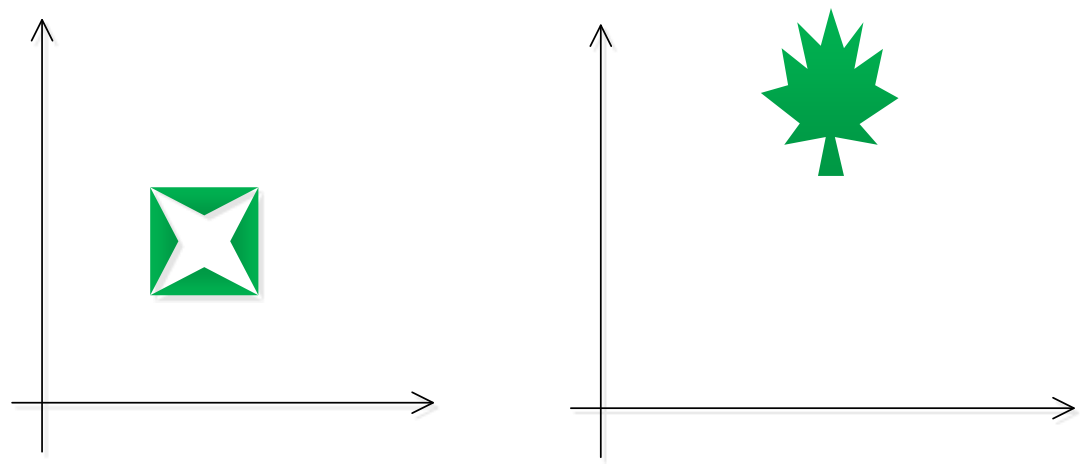

Şekil 7. Sıklaştırma kümeleri $C^{1}$ (solda) ve $C^{2}$ (sağda) 

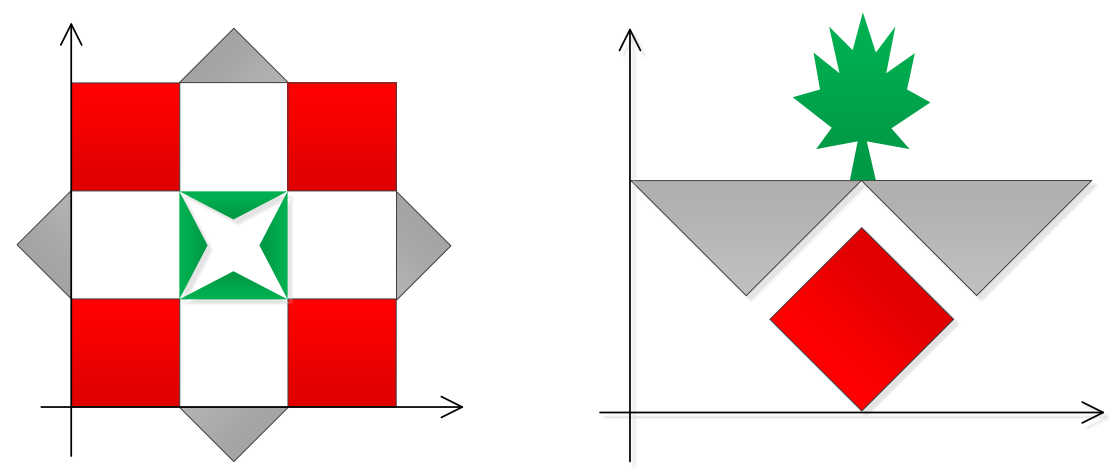

Şekil 8. Metrik uzaylar arasındaki büzülme ve sıklaştırma dönüşümleri
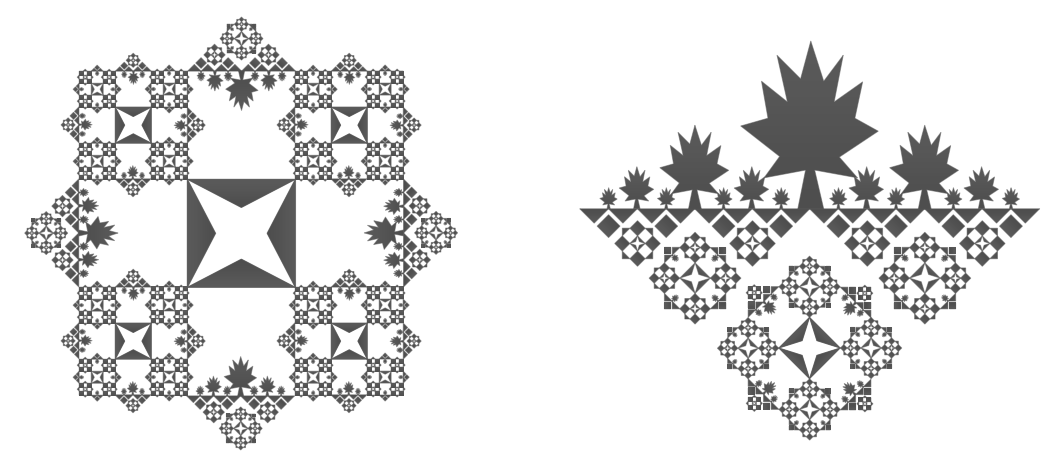

Şekil 9. İterasyonun dördüncü adımında ilgili SÇYFS'nin atraktörleri: $A^{1}$ (solda), $A^{2}$ (sağda)

\section{Sıklaştırma Fonksiyonlu ÇYFS'nin Hausdorff Boyutu}

Bu bölümde, tanımladığımız SÇYFS'lerin atraktörlerinin Hausdorff boyutu ile ilgileneceğiz. Snigireva ve Fraser, [9] ve [10] çalışmalarında sıklaştırma fonksiyonlu bir yinelemeli fonksiyon sisteminin atraktörlerinin Hausdorff boyutu (hatta sayılabilir kararlılık özelliğine sahip herhangi bir boyut kavramı) ve fraktal boyutu ile ilgili çalışmalar yapmışlardır. Snigireva bir klasik SFYFS'nin atraktörünün Hausdorff boyutunun, ilgili sıklaştırma kümesinin Hausdorff boyutu ile, sıklaştırma kümesi olmadan elde edilen atraktörün Hausdorff boyutunun maksimumuna eşit olduğunu göstermiş, dahası üst ve alt fraktal boyut ile ilgili de bazı sonuçlar elde etmiştir.

$\left\{X^{u} ;\left(f_{e}\right)_{e \in E}\right\}$ sistemi $\mathcal{G}=(V, E, r(e))$ Mauldin-Williams çizgesini gerçekleyen bir ÇYFS olmak üzere $\left\{X^{u} ;\left(f_{e}\right)_{e \in E}, w_{u}\right\}$ SÇYFS'ni düşünelim. Her $u \in V$ için $C^{u}$ (sıklaştırma) kümelerinin $X^{u}$ içinde yaşayan (dolayısıyla $A^{u}$ atraktörünün içinde bulunan) bütün küçük kopyalarının birleşimi olan küme

$$
T^{u}=C^{u} \cup \bigcup_{v \in V, i(\alpha)=u} f_{\alpha}\left(C^{v}\right)
$$

şeklinde ifade edilebilir. $\left(i(\alpha)=e_{1}=u\right.$ şartının sadece $u$ 'dan başlayan bütün yollara izin verdiğini hatırlayalım. $f_{\alpha}: X^{\mathrm{v}} \rightarrow X^{u}$ olacağından sıklaştırma kümelerinin sadece $X^{u}$ 'daki kopyaları dikkate alınmış olur.) 
Yardımcı Teorem 3.1 Her $u \in V$ için

$$
T^{u}=C^{u} \cup \bigcup_{v \in V} \bigcup_{e \in E_{u v}} f_{e}\left(T^{v}\right)
$$

eşitliği geçerlidir.

Kanıt. $T^{u}$ 'nun tanımı kullanılarak

$$
\begin{aligned}
C^{u} \cup \bigcup_{v \in V} \bigcup_{e \in E_{u v}} f_{e}\left(T^{v}\right) & =C^{u} \cup \bigcup_{v \in V} \bigcup_{e \in E_{u v}} f_{e}\left(C^{v} \cup \bigcup_{w \in V, i(\alpha)=v} f_{\alpha}\left(C^{w}\right)\right) \\
& =C^{u} \cup \bigcup_{v \in V} \bigcup_{e \in E_{u v}}\left[f_{e}\left(C^{v}\right) \cup f_{e}\left(\bigcup_{w \in V, i(\alpha)=v} f_{\alpha}\left(C^{w}\right)\right)\right] \\
& =C^{u} \cup \bigcup_{v \in V} \bigcup_{e \in E_{u v}}\left[f_{e}\left(C^{v}\right) \cup \bigcup_{w \in V, i\left(\alpha^{\prime}\right)=u} f_{\alpha^{\prime}}\left(C^{w}\right)\right] \\
& =C^{u} \cup\left[\bigcup_{v \in V} \bigcup_{e \in E_{u v}} f_{e}\left(C^{v}\right)\right] \cup\left[\bigcup_{w \in V, i\left(\alpha^{\prime}\right)=u} f_{\alpha^{\prime}}\left(C^{w}\right)\right]
\end{aligned}
$$

elde edilir. Burada $f_{\alpha^{\prime}}=f_{e} \circ f_{\alpha}$ olduğuna ve $\left|\alpha^{\prime}\right|>1$ olduğuna dikkat ediniz. Son birleşimdeki ikinci terim, $\forall v \in V$ için $C^{v}$ kümesinin 1 uzunluklu mümkün bütün yollara karşılık gelen fonksiyonlar altında $X^{u}$ içinde yaşayan kopyalarıdır. Üçüncü terim ise uzunluğu 1'den büyük olan bütün yollara karşılık gelen fonksiyonlar altındaki kopyalardır. Sonuç olarak son ifadedeki birleşim tam olarak $T^{u}$ kümesini vermektedir.

Yardımcı Teorem 3.2 Her $u \in V$ için $\overline{T^{u}}=A^{u}$ şeklindedir.

Kanıt. Yardımcı Teorem 3.1 kullanılarak,

$$
\begin{aligned}
\overline{T^{u}} & =\overline{C^{u} \cup \bigcup_{v \in V} \bigcup_{e \in E_{u v}} f_{e}\left(T^{v}\right)} \\
& =\overline{C^{u}} \cup \overline{\bigcup_{v \in V} \bigcup_{e \in E_{u v}} f_{e}\left(T^{v}\right)}
\end{aligned}
$$




$$
\begin{aligned}
& =C^{u} \cup \overline{\bigcup_{v \in V} \bigcup_{e \in E_{u v}} f_{e}\left(T^{v}\right)} \\
& =C^{u} \cup \bigcup_{v \in V} \bigcup_{e \in E_{u v}} \overline{f_{e}\left(T^{v}\right)} \\
& =C^{u} \cup \bigcup_{v \in V} \bigcup_{e \in E_{u v}} f_{e}\left(\overline{T^{v}}\right)
\end{aligned}
$$

bulunur. Ancak, Teorem 2.2'de de belirtildiği üzere ilgili eşitliği sağlayan her $u=1,2, \ldots, N$ için boştan farklı tek kompakt küme $A^{u}$ olduğundan, $\overline{T^{u}}=A^{u}$ 'dur.

$\left\{X^{u} ;\left(f_{e}\right)_{e \in E}, w_{u}\right\}$ SÇYFS'nin atraktörleri $\left(A^{u}\right)_{u \in V}$ şeklinde olmak üzere, sıklaştırma fonksiyonları kullanmadan var olan klasik $\left\{X^{u} ;\left(f_{e}\right)_{e \in E}\right\}$ ÇYFS'nin atraktörlerini de $\left(A_{\emptyset}^{u}\right)_{u \in V}$ şeklinde gösterelim.

Yardımcı Teorem 3.3 Her $u \in V$ için $A^{u}=A_{\varnothing}^{u} \cup T^{u}$ eşitliği sağlanır.

Kanıt. Klasik durum için [9] çalışmasında, $A$ ve $A_{\varnothing}$ sırasıyla SFYFS ve YFS'nin atraktörleri ve $T$ de $C$ sıklaştırma kümesi ve $C$ 'nin YFS'deki tüm fonksiyonların mümkün bütün bileşkeleri altındaki görüntülerinin birleşimini gösteren küme olmak üzere $A=A_{\emptyset} \cup$ $T$ olduğu kanıtlanmıştır.

Benzer argümanlarla, Yardımcı Teorem 3.1 ve Yardımcı Teorem 3.2 yardımı ile her $u \in V$ için $A^{u}=A_{\varnothing}^{u} \cup T^{u}$ kolayca görülür.

Örnek 3.1. Örnek 2.1'de verilen SÇYFS'nin atraktörleri, bu atraktörlerdeki sıklaştırma kümelerinin ilgili atraktörlerdeki tüm kopyaları ve Örnek 1.3'de verilen sıklaştırma fonksiyonu kullanılmadan elde edilen atraktörler Şekil 10’da gösterilmiştir.
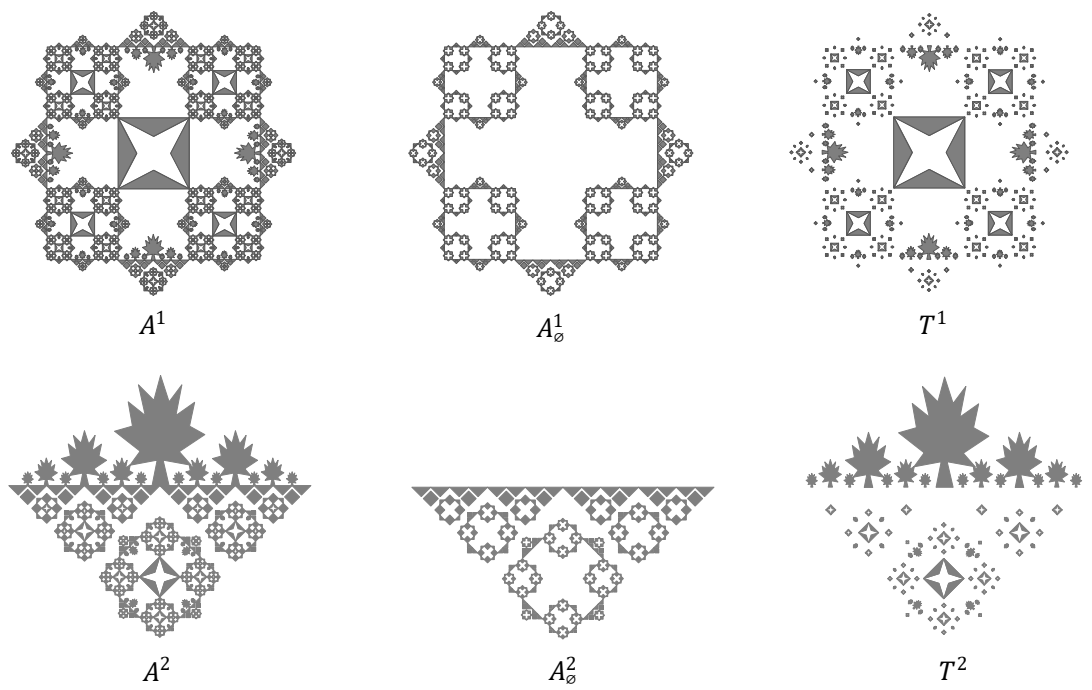

Şekil 10. Örnek 2.1'de verilen SÇYFS için (4.adımda) $A^{u}, A_{\varnothing}^{u}$ ve $T^{u}$ kümeleri 
$\left\{A_{i}\right\}_{i \in I}$ sayllabilir bir küme dizisi olsun. $\operatorname{dim}_{H} A, A$ kümesinin Hausdorff boyutunu göstermek üzere

$$
\operatorname{dim}_{H}\left(\bigcup_{i \in I} A_{i}\right)=\sup _{i \in I}\left\{\operatorname{dim}_{H}\left(A_{i}\right)\right\}
$$

eşitliği geçerlidir (bu özelliğe genel olarak sayılabilir kararlılık özelliği denir).

Teorem $3.4\left\{X^{u} ;\left(f_{e}\right)_{e \in E}\right\}$ kuvvetli-bağlantılı bir $\mathcal{G}=(V, E, r(e))$ Mauldin-Williams çizgesini gerçekleyen bir ÇYFS olmak üzere $\left\{X^{u} ;\left(f_{e}\right)_{e \in E}, w_{u}\right\}$ SÇYFS verilsin. Her $u \in V$ için ilgili sistemlerin atraktörlerini sırasıyla $A_{\varnothing}^{u}$ ve $A^{u}$ ile gösterelim. Bu durumda

eşitliği geçerlidir.

$$
\operatorname{dim}_{H} A^{u}=\max \left\{\operatorname{dim}_{H} A_{\varnothing}^{u}, \max _{v \in V}\left\{\operatorname{dim}_{H} C^{v}\right\}\right\}
$$

Kanıt. Yardımcı Teorem 3.3 kullanılırsa her $u \in V$ için,

$$
A^{u}=A_{\varnothing}^{u} \cup T^{u}=A_{\varnothing}^{u} \cup C^{u} \cup \bigcup_{v \in V, i(\alpha)=u} f_{\alpha}\left(C^{v}\right)
$$

şeklindedir. Bu eşitliğin yanında, $\operatorname{dim}_{H}$ sayılabilir kararlllık özelliğine sahip olduğundan

$$
\begin{aligned}
\operatorname{dim}_{H} A^{u} & =\max \left\{\operatorname{dim}_{H} A_{\varnothing}^{u}, \operatorname{dim}_{H} C^{u}, \operatorname{dim}_{H}\left(\bigcup_{v \in V, i(\alpha)=u} f_{\alpha}\left(C^{v}\right)\right)\right\} \\
& =\max \left\{\operatorname{dim}_{H} A_{\varnothing}^{u}, \operatorname{dim}_{H} C^{u} \sup _{v \in V, i(\alpha)=u}\left\{\operatorname{dim}_{H} f_{\alpha}\left(C^{v}\right)\right\}\right\}
\end{aligned}
$$

elde edilir. Herhangi bir kümenin bir büzülme dönüşümü altındaki görüntüsünün Hausdorff boyutu kendisinin Hausdorff boyutundan küçük veya eşit, $f_{\alpha}$ dönüşümleri büzülme dönüşümü ve ilgili çizge kuvvetli-bağlantılı (dolayısıyla herhangi iki metrik uzay arasında (yönlü) bir fonksiyon ve bu nedenle herbir atraktör içinde bütün sıklaştırma kümelerinin kopyaları var) olduğundan dolayı

$$
\operatorname{dim}_{H} A^{u}=\max \left\{\operatorname{dim}_{H} A_{\varnothing}^{u}, \max _{v \in V}\left\{\operatorname{dim}_{H} C^{v}\right\}\right\}
$$

eşitliği elde edilir.

$\left\{X^{u} ;\left(f_{e}\right)_{e \in E}\right\} \quad$ kuvvetli-bağlantılı bir $\mathcal{G}=(V, E, r(e))$ Mauldin-Williams çizgesini gerçekleyen bir ÇYFS ise, bu sistemin atraktörlerin herbirinin boyutu birbirine eşittir. $\mathrm{Bu}$ atraktörlerinin boyutlarının hesaplanması ayrı ve önemli bir problemdir (detay için bkz. [4]). Ama bu sisteme siklaştırma fonksiyonu eklendiğinde yani sabit kompakt kümelerle atraktörler sıklaştırıldığında elde edilen atraktörlerin boyutları, bu sıklaştırma kümelerinin boyutları ile, ilgili ÇYFS sisteminin atraktörlerinin boyutlarının maksimumu olarak hesaplanir. 


\section{Araştırmacıların Katkı Oranı Beyanı}

Fatma Diğdem KOPARAL: Araştırma, Kavramsallaştırma, Yazılım, İnceleme ve Düzenleme, Orijinal Taslak Yazımı.

Yunus ÖZDEMIR: Araştırma, Kavramsallaştırma, Yazılım, İnceleme ve Düzenleme, Orijinal Taslak Yazımı.

\section{Destek ve Teşekkür Beyanı}

Bu çalışma Anadolu Üniversitesi tarafından 1605F473 numaralı araştırma projesi kapsamında desteklenmiştir.

\section{Çatışma Beyanı}

$\mathrm{Bu}$ çalışmanın yazarları olarak herhangi bir çatışma beyanımız bulunmadığını bildiririz.

\section{Etik Kurul Onayı ve/veya Aydınlatılmış Onam Bilgileri}

Bu çalışmanın yazarları olarak herhangi bir etik kurul onayı ve/veya aydınlatılmış onam bilgileri beyanımız bulunmadığını bildiririz.

\section{Kaynakça}

[1] M. F. Barnsley, Fractals Everywhere. New York: Academic Press, 1988.

[2] M. F. Barnsley and S. Demko, "Iterated function systems and the global construction of fractals," Proc. $R$. Soc. Lond. Ser. A, 399, 243-275, 1985.

[3] J. E. Hutchinson, "Fractals and self-similarity," Indiana Univ. Math. J., 30, 713-747, 1981.

[4] G. Edgar, Measure, Topology and Fractal Geometry. New York: Springer, 2008.

[5] R. D. Mauldin and S.C. Williams, "Hausdorff dimension in graph directed constructions," Trans. Amer. Math. Soc., 309(2), 811-829, 1988.

[6] S. Baker, J. M. Fraser, and A. Máthé, "Inhomogeneous self-similar sets with overlaps," Ergod. Theor. Dyn. Syst., 39(1), 1-18, 2019.

[7] Z. G. Yu, V. V. Anh, and K. S. Lau, "Hausdorff dimension of random fractals with overlaps," Bull. Aust. Math. Soc., 65(2), 315-328, 2002.

[8] Y. Li, "Fractal dimension estimation for color texture images," J. Math. Imaging Vision, 62, 37-53, 2020.

[9] N. Snigireva, "Inhomogeneous Self-similar Sets and Measures," Ph.D Dissertation, University of St Andrews, 2008.

[10] J. M. Fraser, "Inhomogeneous self-similar sets and box dimensions," Studia Mathematica, 213(2), 133$155,2012$.

[11] M. Holland and Y. Zhang, "Dimension results for inhomogeneous Moran set constructions," Dynamical Systems, 28(2), 222-250, 2013.

[12] G. Edgar and J. Golds, "A fractal dimension estimate for a graph-directed iterated function system of non-similarities," Indiana Univ. Math. J., 48(2), 429-447, 1999.

[13] P. R. Massopust, Fractal functions, fractal surfaces, and wavelets. 2nd edition, Amsterdam: Academic Press, 2016. 